\title{
Unsupervised Human Detection with an Embedded Vision System on a Fully Autonomous UAV for Search and Rescue Operations
}

\author{
Eleftherios Lygouras ${ }^{1, *}$, Nicholas Santavas ${ }^{1}$ (D), Anastasios Taitzoglou ${ }^{1}$ (D), \\ Konstantinos Tarchanidis ${ }^{2}$, Athanasios Mitropoulos ${ }^{2}$ and Antonios Gasteratos ${ }^{1}$ (D) \\ 1 Department of Production and Management Engineering, Democritus University of Thrace, \\ 67100 Xanthi, Greece \\ 2 Department of Petroleum, Natural Gas and Mechanical Engineering, Eastern Macedonia and Thrace \\ Institute of Technology, 65404 Kavala, Greece \\ * Correspondence: elygoura@ee.duth.gr; Tel.: +30-6931-087-842
}

Received: 20 June 2019; Accepted: 12 August 2019; Published: 14 August 2019

\begin{abstract}
Unmanned aerial vehicles (UAVs) play a primary role in a plethora of technical and scientific fields owing to their wide range of applications. In particular, the provision of emergency services during the occurrence of a crisis event is a vital application domain where such aerial robots can contribute, sending out valuable assistance to both distressed humans and rescue teams. Bearing in mind that time constraints constitute a crucial parameter in search and rescue (SAR) missions, the punctual and precise detection of humans in peril is of paramount importance. The paper in hand deals with real-time human detection onboard a fully autonomous rescue UAV. Using deep learning techniques, the implemented embedded system was capable of detecting open water swimmers. This allowed the UAV to provide assistance accurately in a fully unsupervised manner, thus enhancing first responder operational capabilities. The novelty of the proposed system is the combination of global navigation satellite system (GNSS) techniques and computer vision algorithms for both precise human detection and rescue apparatus release. Details about hardware configuration as well as the system's performance evaluation are fully discussed.
\end{abstract}

Keywords: unmanned aerial vehicles (UAVs); search and rescue (SAR) missions; human detection; deep learning

\section{Introduction}

UAV technology demonstrates unprecedented levels of growth and influence in various scientific fields and research areas. Owing to their tremendous advantage of being able to automatically perform complex tasks and missions, the application domains of such vehicles are ceaselessly increasing. One of the most challenging application areas of such aerial robots is SAR missions, as crisis events may occur at any given moment and over all kinds of places [1]. The success rate of such procedures is highly depended on the time needed for first responder awareness that a human life is in imminent danger. The faster these operations are designed and executed, the greater the chances of success they get. Thus, this application area can greatly benefit from aerial robots capable of detecting and rescuing humans facing dire straits in, mainly but not only, maritime critical events [1-4]. Rescue UAVs are valuable during crisis management due to their maneuverability and versatility. These vehicles are capable of speeding up rescue missions while improving their efficacy. Additionally, with a view to increase the victim's survival time, these flying robots can transport rescue apparatus much faster than rescue crews [5]. Moreover, such vehicles should be plausibly considered as a robust helping hand 
to the first responder teams as they are beneficial in terms of providing aerial visibility of the critical event while ensuring rescue worker safety [6].

The utilization of UAVs as a complement of search and rescue activities has gotten continuously increasing support by researchers and organizations around the globe. Numerous rescue systems including teleoperated UAVs have been proposed to establish robotic assistance in emergency management $[7,8]$. Taking into account the emerging necessity for an utterly autonomous rescue UAV, an aerial rescue support system was proposed in our prior research $[9,10]$. With the aim to navigate and operate fully autonomous ROLFERs (Robotic Lifeguard For Emergency Rescue), our aspiration is to provide instantaneous emergency services to humans in peril during a maritime crisis occurrence while boosting rescue team performance by significantly minimizing their workload. Detecting distressed humans from the developed aerial rescue system has mainly relied on GNSS techniques. Notable research has been conducted for the GNSS precise point positioning (PPP) accuracy of portable devices such as smartphones, tested in both post-processing and real-time modes [11-13]. The results of their positioning performance assessment compared to the geodetic receivers' ones indicate that the utilization of multi GNSS systems data can effectively improve positioning performance, which is essential for missions in marine environments. Besides, due to the lack of commercially available wearable, waterproof, and portable devices with acceptable accuracy, there is a pressing need to enhance the system's operational performance through the precise location of the victim. The obvious solution to employ computer vision techniques to solve this problem is not straightforward, owing to several restrictions in conventional classifier accuracy and precision. That have been said, contemporary deep learning techniques [14] can overcome such issues, yet their complexity makes their implementation on a portable system, where hardware means should be rather austere, a cumbersome procedure.

The paper in hand proposes an embedded system implementing a deep learning architecture trained for the identification and location of swimmers. Thus, we address the problem of precise open water human detection by conducting real-time recognition onboard a rescue hexacopter, as well as the accurate transportation and release of the airborne rescue apparatus. The main novelty of this research banks on the collection of a novel dataset, suitable for training state-of-the-art computer vision algorithms, the implementation of deep learning algorithms on an embedded system suitable for a medium payload carrying capability hexacopter, and their seamless integration with an utterly autonomous search and rescue system. In particular, in order to obtain an adequate size dataset for the chosen model, the majority of the training dataset used in this research are images extracted by videos captured by the authors. Humans swimming in open waters were filmed by means of a UAV flying at several altitudes. The annotated dataset is freely available to any member of the community. The remainder of this paper is structured in the following manner. Section 2 provides an overview of the conducted related research. Theory and approach of our work are presented in Section 3. The system's human detection procedure is described in Section 4. In Section 5 we provide some crucial information about the hardware configuration, as well as the system's implementation details. Conclusions are drawn in Section 6.

\section{Related Research}

Object detection has been a broad field of study in computer vision over the past decades. The superior performance of deep learning techniques in detecting and classifying objects in images has led to their widespread adoption in a wide variety of applications [15]. Several approaches and architecture for object detection, segmentation and action recognition throughout videos have been proposed in the literature, including many variations of convolutional neural networks (CNNs) such as temporal convolutional networks (T-CNN) [16-18], 3D CNNs [19-21] and many more.

A noteworthy overview of deep learning methods and applications combined with UAVs technology has been conducted in [22]. The main challenges, as well as the performance and limitations of such developments were discussed in detail. Furthermore, several unsolved challenges for efficient performance of unsupervised learning were pointed out. Yet, the selection and the adjustment of the operational parameters for the training of CNNs capable of performing object detection are of vital 
importance due to the high dependency of a model's reliability and accuracy to such procedures [23]. An approach for the implementation of such systems has been presented in [24]. Data collection, deep networks architecture and training stages for an embedded processing platform, performing vehicle detections through aerial images, are highlighted.

Bearing in mind UAV competence in providing precious aerial visual information, the implementation of such techniques on aerial systems is an increasing trend in various research fields [25-29]. Issues related to sensing and connectivity of sensors for the assisted or even the autonomous navigation of UAVs, as well as the reliability and the maintenance analysis of such vehicles have been extensively discussed in [30,31]. Moreover, the adaption of camera parameters, as well as motion parameters estimation based on the integration of video measurements and data obtained by UAVs, have always to be seriously taken into account [32]. The relentless motivation for image classification tasks based on deep learning and aerial images captured by UAVs has led to an abundance of research including vehicles [24,33,34], aerial vehicles [35-39], roads [40], buildings [41,42], cracks [43], birds [44], cattle [45], and wilt [46] detection. Another remarkable approach for object detection in very high-resolution aerial images has been proposed in [47]. Using a one-stage densely connected feature pyramid network, the proposed model tackles the problem of target object detection on different scales. Assessment on two datasets in terms of computation time and mean average precision $(\mathrm{mAP})$ was also provided. A primordial task for researchers dealing with computer vision and UAVs, is the precise autonomous landing of such vehicles on both static or moving targets [48-51]. The detection of a desired target and the landing of the aerial vehicles on a desired target totally depend on onboard sensing and computing, without requiring external infrastructure or prior information of the target's location. Nevertheless, human identification in videos or images is the most crucial task across a variety of scientific fields. Thus, real-time human detection from an aerial perspective based on deep learning approaches is an extremely active research area [52-54]. Consequently, when it comes to crisis events, a dramatic demand for rapid and accurate detection of an individual whose life is at risk arises, as the provision of instantaneous rescue services is urgent for his/her survival.

Search and rescue operations can greatly benefit by deep learning techniques applied in UAVs, as such developments exhibit high performance in detecting and precisely locating humans in peril while decreasing the response time that rescuers would need to do so. Nevertheless, the separation of crowded and non-crowded regions for safe UAV flight is apparent. The research in [55] proposes a novel method for enhancing the definition of non-fly zones for crowd avoidance by utilizing deep CNNs. A notable approach for the detection of human survivors in disaster environments has been conducted in [56]. The proposed system detects human existence by applying a feed-forward neural network, acquiring a small amount of data with high detection accuracy. In [57] a method for SAR mission assistance in case of avalanche debris was proposed. Furthermore, an innovative, state-of-the-art technique for the estimation of snow-field parameters has been presented in [58]. The unmanned aerial system (UAS)-based photogrammetry method significantly reduces the data collection time and health risks associated with avalanches. A system composed of a pre-processing method, a pre-trained CNN, a trained linear support vector machine (SVM) classifier and a post-processing method for human detection rate increase in images captured by a UAV, is analytically presented there. The performance of accurate real-time object detection on-board a UAV has been outlined in [59]. Capable of accurately detecting objects during emergency situations, the developed system critically feeds a decision support warning system for situational awareness. In [60], a notable approach for victim detection in complex environments based on the color and depth data captured from onboard UAV sensors was proposed. The implemented reactive control system's validation, including both real flights and simulations, exhibited interesting results about multiple individuals tracking even after long-term disappearances. Last but not least, a project for industrial firefighter performance enhancement has been presented in [61]. Aiming to reduce their cognitive load, firefighters can benefit from mission-guided control and enhanced autonomy of the system in known indoor and outdoor environments by assigning mission goals with priorities to drones. 
In conclusion, advances in deep learning have attracted researchers' interest for applying deep reinforcement learning methods to rescue robots for their autonomous navigation [62], efficient exploration of various unknown environments [63], SAR purposes in indoor environments [64], and for target recognition and interaction between aerial robots and targets.

\section{Deep Learning}

Deep learning methods and techniques have been getting a dramatically increased popularity among the research areas of neural networks and artificial intelligence. Their increased processing capabilities, the huge amount of data used for training, and the recent advances in machine learning and signal processing are the main reasons for being held in high regard by researchers [15]. Their superior capabilities in solving complex tasks on many facets of information processing have led to their breathtaking impact on numerous application areas. Autonomous robotics applications are a bold example of the suitability of deep learning techniques utility and their huge beneficial potential. The combined use of such methods and UAV technologies have presented a drastic increase recently, due to the fact that such techniques are capable of extracting exceptionally useful information from aerial images [22]. Convolutional neural networks are state-of-the-art in the advanced application of deep learning for a broad range of tasks such as image or video processing, speech recognition, text digitization, and more. CNNs utilization for image-related purposes is the most popular application domain of this specific type of neural network algorithm. Their marvelous capability of detecting what an image is or what an image contains, or even automatically generating captions for images, has impressively increased their popularity and their feasibility in the computer vision field $[65,66]$.

YOLO $[67,68]$ is an open source state-of-the-art object detection system for real-time processing. Using a totally different approach, YOLO has several advantages, compared to prior region object detection systems and classification networks, in the way it performs detection and prediction. Region proposal classification networks perform detection by applying the model to an image with multiple predictions in various image regions and scales. High scoring regions are considered as detections, yet YOLO uses a one-stage detector strategy and its architecture is similar to a fully convolutional neural network (FCNN). Specifically, YOLO treats object detection as a regression problem by applying a single $\mathrm{CNN}$ and bounding boxes to the full image, for both the object's classification and localization. The image is divided into regions and predictions are made by bounding boxes weighted by the predicted probabilities. The main YOLO advantage for real-time object detection is the improvement of deep learning-based detection procedure speed.

\section{Human Detection}

Detecting humans in images cropped from a video stream either on land or a marine environment, is a challenging task due to the human body's variable appearance and the wide range of poses it can adopt. Moreover, video footage collected by a UAV differs substantially from images acquired on the ground; therefore the use of standard techniques is not straightforward. The following aspects and assumptions must be considered when designing the image processing algorithm. Firstly, the typical distances from a camera to human bodies of interest are generally larger than in the case of standard "ground" techniques (e.g., office-like environments, surveillance cameras). Additionally, the human footage in a marine environment may differ substantially from a land one. In a common land situation, the whole human silhouette should be visible, yet in a water one it is most likely that only a part of it (i.e., the upper body) is visible. Moreover, should the vehicle be operational during different times of the day, the human detection algorithm must be equally effective under all lighting conditions. Therefore, it is essential for the image processing algorithm to take into account all the aforementioned considerations. A robust feature set that allows the human silhouette to be discriminated clearly, even in cluttered backgrounds under different poses and different illumination conditions, is apparently needed. In this article we study the combination of the open source software routines, libraries and algorithms available today with a low cost vision camera, in order to design 
a system that can effectively detect, localize, and track a human in a marine environment, under all lighting conditions. For this reason, we focus on computational efficient computer vision algorithms; even at the cost of low frame rate.

In order to detect swimmers, each frame of live video captured by the camera onboard the UAV is processed separately by the pipeline. Firstly, the input image is divided into a $S \times S$ grid with $N$ number of cells, as shown in Figure 1 (Left). Should the centre of the object be laid within a grid cell then this specific cell is "responsible" for the object's detection. At this point, each grid cell predicts $B$ bounding boxes as well as confidence scores for those boxes (see Figure 1 (Top)). These confidence scores reflect the confidence of the model that an object is contained in the box, as well as the accuracy of the box that it predicts. Confidence is formally defined as

$$
\operatorname{Conf}(\%)=\left(P_{r}(\text { object }) \times \operatorname{IoU}_{\text {pred }}^{\text {truth }}\right) \times 100 \%
$$

where Conf is the confidence level expressed in a percentage score, $P_{r}$ is the predicted box containing an object, and IoU is the intersection over union between the predicted box and the ground truth.

In the ideal situation where the object is contained within the predicted box, then Conf equals IoU; while should no object exist in the box, Conf equals zero.

Every single bounding box contains 5 predictions, namely: The $(x, y)$ coordinates of the bounding box center, the box dimensions (i.e., width $(w)$ and height $(h))$ and finally the probability of objects' appearance in the bounding box $\left(P_{r}(\right.$ object $\left.)\right)[67,68]$. All these are normalized values (i.e., between 0 and 1 ). Bounding box center coordinates $(x, y)$ are calculated relatively to the bounds of grid's cell, while the width and the height are predicted relatively to the whole image. Thus, the confidence prediction expresses the IoU factor between the ground truth and the prediction box.

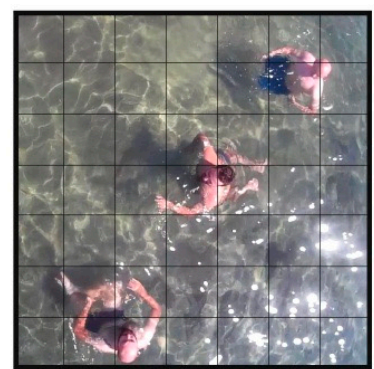

$S \times S$ grid on input
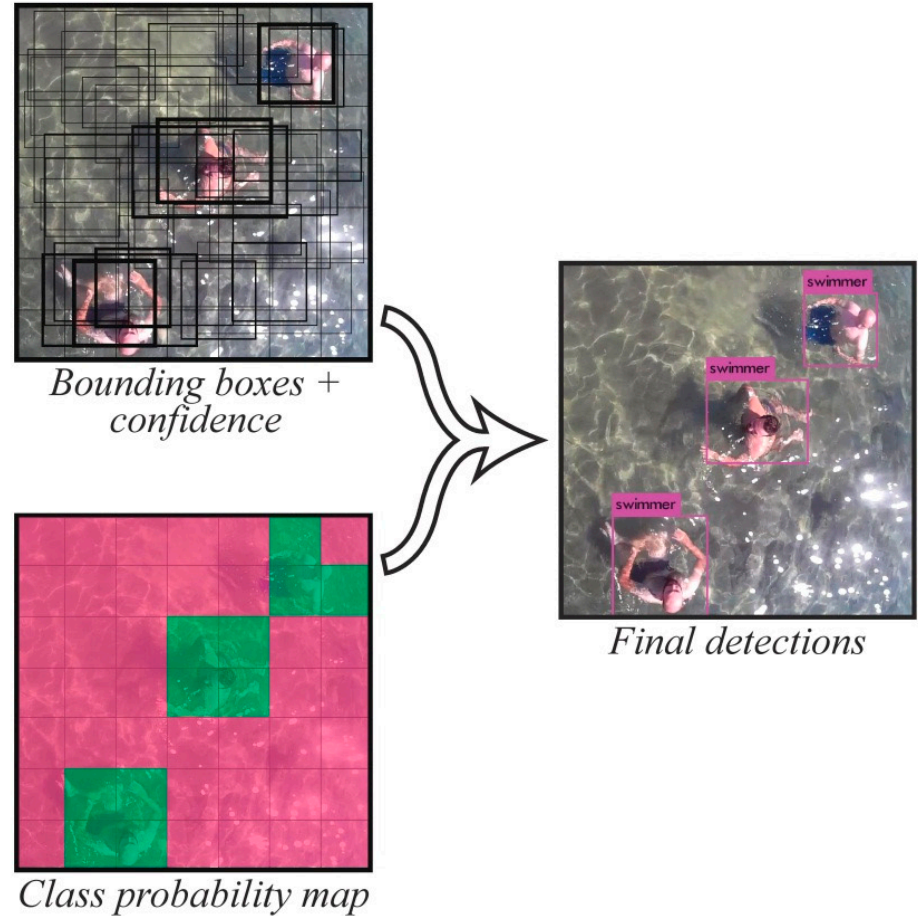

Final detections

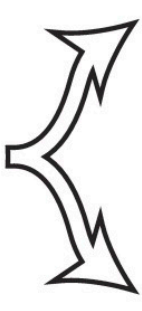

Class probability map

Figure 1. Image captured by the rescue unmanned aerial vehicle (UAV): (Left) divided into equally $S \times$ $S$ sized grid cells, (Top and Bottom) predicting $B$ bounding boxes, confidence scores and $C$ conditional class probabilities, (Right) leading to final encoded detections.

Moreover, each grid cell predicts $C$ conditional class probabilities $\left(P_{r}(\right.$ ClassijObject $\left.)\right)$, which are affected by the grid cell containing an object (see Figure 1 (Bottom)). Previous YOLO versions apply a softmax function to convert scores into probabilities with sum equal to 1. Instead, YOLOv3 [69] 
uses multi-label classification by replacing the softmax function with independent logistic classifiers in order to calculate the probability of an input belonging to a specific label. Thus, the model makes multiple predictions across different scales, with higher accuracy, regardless of the predicted object's size. Finally, the network consists of two main parts - the base model, which operates as feature extractor and the resulting tensor, which encodes the bounding box, the probability of object's existence in the cell (objectness), and the class predictions. The final output of the whole procedure (Figure 1 (Right)) is an encoded $S \times S \times\left(B^{*} 5+C\right)$ tensor where:

$S \times S:$ is the sized grid cells dimensions

$B$ : is the number of bounding boxes and

$C$ : is the number of networks' labeled classes

During the training process, the loss function of end-to-end model should simultaneously address both classification and detection issues, which dictates the learning and the testing accuracy of the detectors. In YOLO, the computation of each prediction's loss is accomplished by using the sum-squared error between the ground truth and the predictions. Thus, the loss function consists of three individual losses:

- Classification Loss: Errors in the prediction's accuracy

- Localization Loss: Errors between predicted boundary box and ground truth

- Confidence Loss: Errors in the object's appearance in the box

The model's architecture used in this paper is the Tiny YOLO V3 [69]. Comprising an improvement of YOLO, Tiny YOLO v3 treats detection somewhat differently by predicting boxes on two different scales while features are extracted from the base network. Its higher performance compared to YOLO was the main reason for its selection.

The model's architecture consists of thirteen convolutional layers with an input size of $416 \times 416$ images, as shown in Figure 2. Due to the limited available amount of dataset, we used transfer-learning for a pre-trained model in order to train a CNN to detect swimmers. The model was trained using COCO dataset [70] and our "swimmers" dataset (https://robotics.pme.duth.gr/swimmers_dataset). The model's training was accomplished by initializing and fixing the depth size of the last convolutional layer's pre-trained weights. Then, we further trained the model with a hand-labelled dataset of open waters swimmers. The model's achieved mAP was approximately $67 \%$.

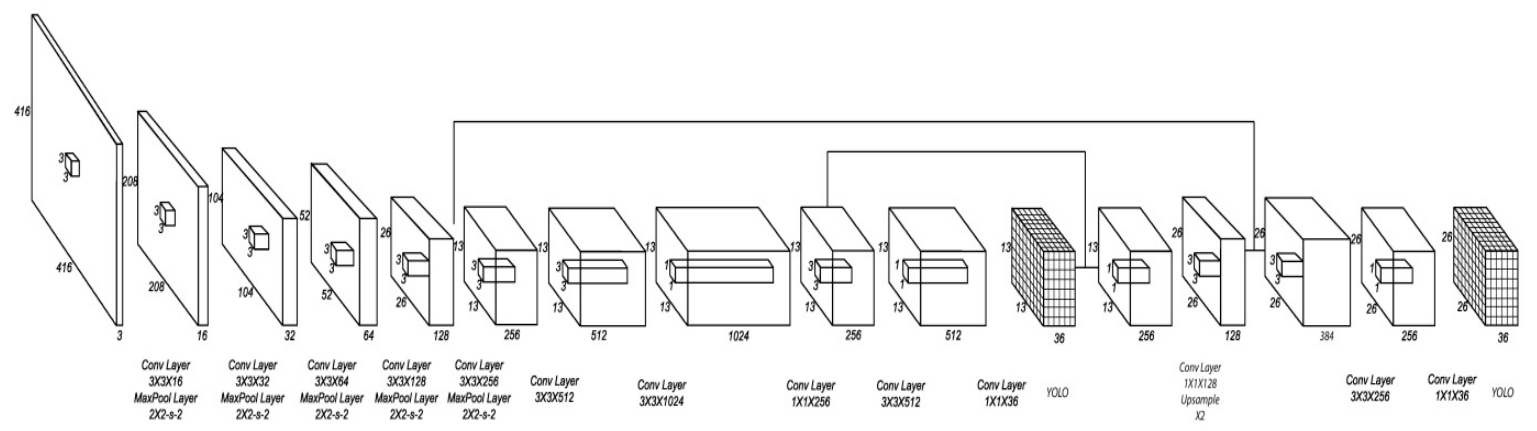

Figure 2. Convolutional neural networks $(\mathrm{CNN})$ model's architecture.

The relation of $\mathrm{mAP}$ to the iteration number of the utilized model is depicted in Figure 3. As it is shown, loss function rapidly decreased as iteration number increased. This provides a nice close view of all stages of the network's learning process. Moreover, the classification accuracy on the test data also changes relatively to the number of iterations. As can be seen, in the first 3000 iterations, there is a big jump in the network's accuracy to approximately 55\%. Next, the accuracy gradually improves till 35,000 iterations, reaching 67\%. Then, the learning process gradually slows down. Finally, at approximately iteration number IN $=44,000$, the classification accuracy fairly stops improving. Later 
iterations merely see small stochastic fluctuations near the value of the accuracy at IN $=44,000$. This means that the network ceases learning at this point, so we concluded that it was trained and after a number of iterations, started overfitting.

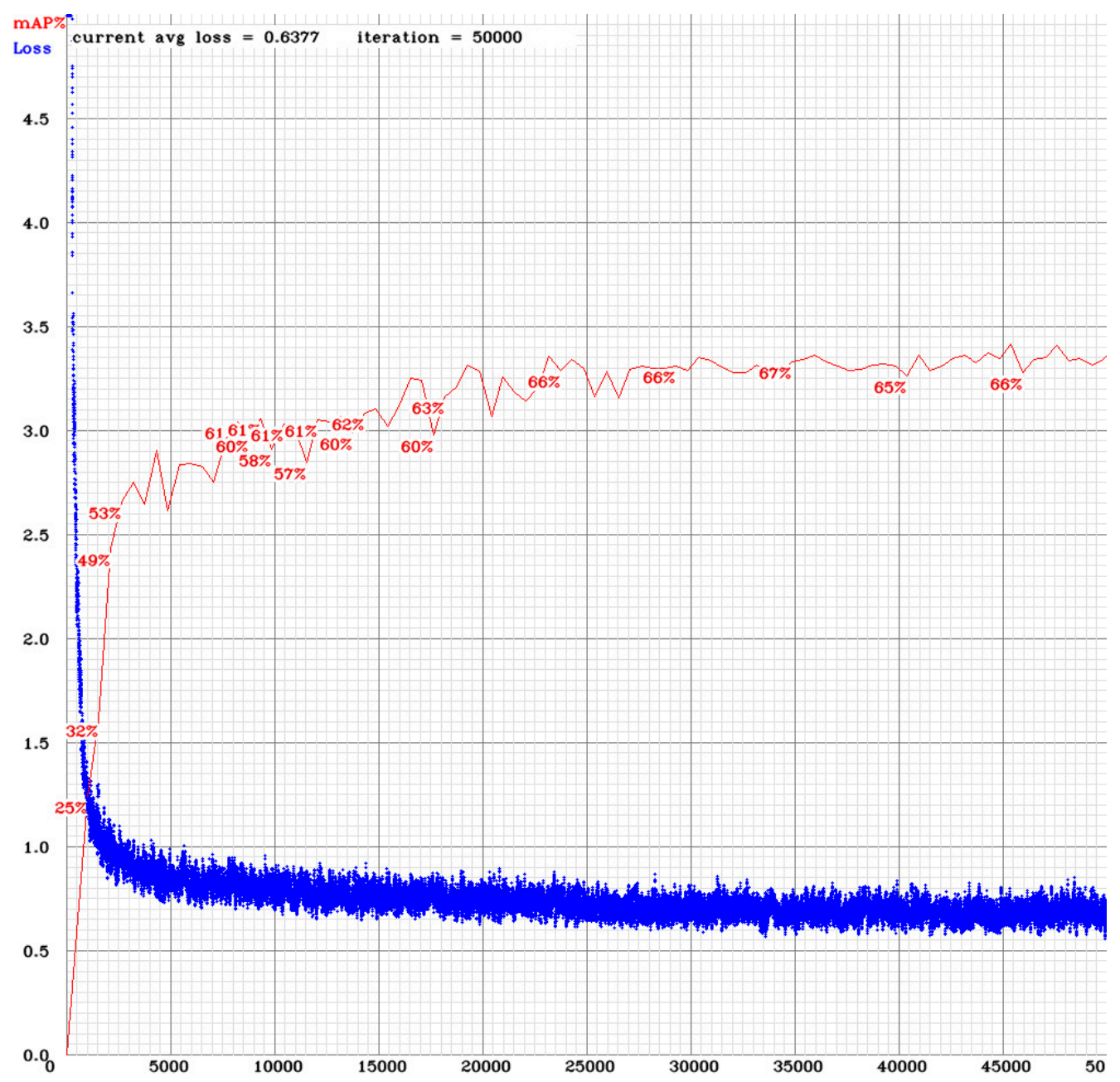

Figure 3. Neural Network's training performance curve.

Concerning the training dataset, we collected from the internet as many swimmers' images as possible, since there was no akin dataset available. Because of the low number of images collected, we were forced to create our own dataset, by manually flying a drone ashore and recording videos containing swimmers. Frames were recorded by means of a GoPro camera, in full HD resolution $(1920 \times 1080$ pixels), and in different angles and lighting conditions. After the network's first training attempt, we observed a significant number of false positive detections, such as boats. Thus, in order to deal with the low "recall" figures, we executed another run of image collection, in the same magnitude as the previously obtained images, yet containing objects that the network false detected. In this way we managed to increase recall and mAP. Eventually, a total of 4500 images were collected. For increased robustness and improved recall values, negative images at 1:1 ratio were also included. The total training dataset of the proposed CNN model was 9000 images, while $10 \%$ of them were kept as validation data. The dataset was split by $90 \%$ as training and $10 \%$ as validation dataset, i.e., the volume of the training set was 8100 images. All subjects were informed and gave their consent to conduct the experiments. The images gathered differed in terms of number of humans in the scene, human characteristics, image scales and background, lighting conditions, resolution, and composition. Finally, a total of 4500 images were collected. Last, with the aim to create our unique training dataset, 
all swimmer images had to be labeled. Thus, an open-source labelling tool was used in order to create ground truth bounding boxes in each of these images.

Although the video recording resolution was full HD, the network was multi-scaled trained. Because of the architecture's nature, which was fully convolutional, every layer could be resized on the fly. During the training procedure, the model randomly downsampled the initial resolution (we chose $608 \times 608$ ) of every 10 batches by a factor of 32 . This regime forces the network to learn to predict across a variety of input dimensions [68]. In the stage of inference, the network's input resolution was set to $416 \times 416$ pixels and the results of mAP were calculated using this resolution. The YOLO algorithm resizes the training dataset to the initial resolution, as mentioned above. Due to the multi-scale training procedure, there is no need to change the initial resolution during training. The only resolution change that matters is the one along inference, because of the different performance results as depicted in Figure 4.

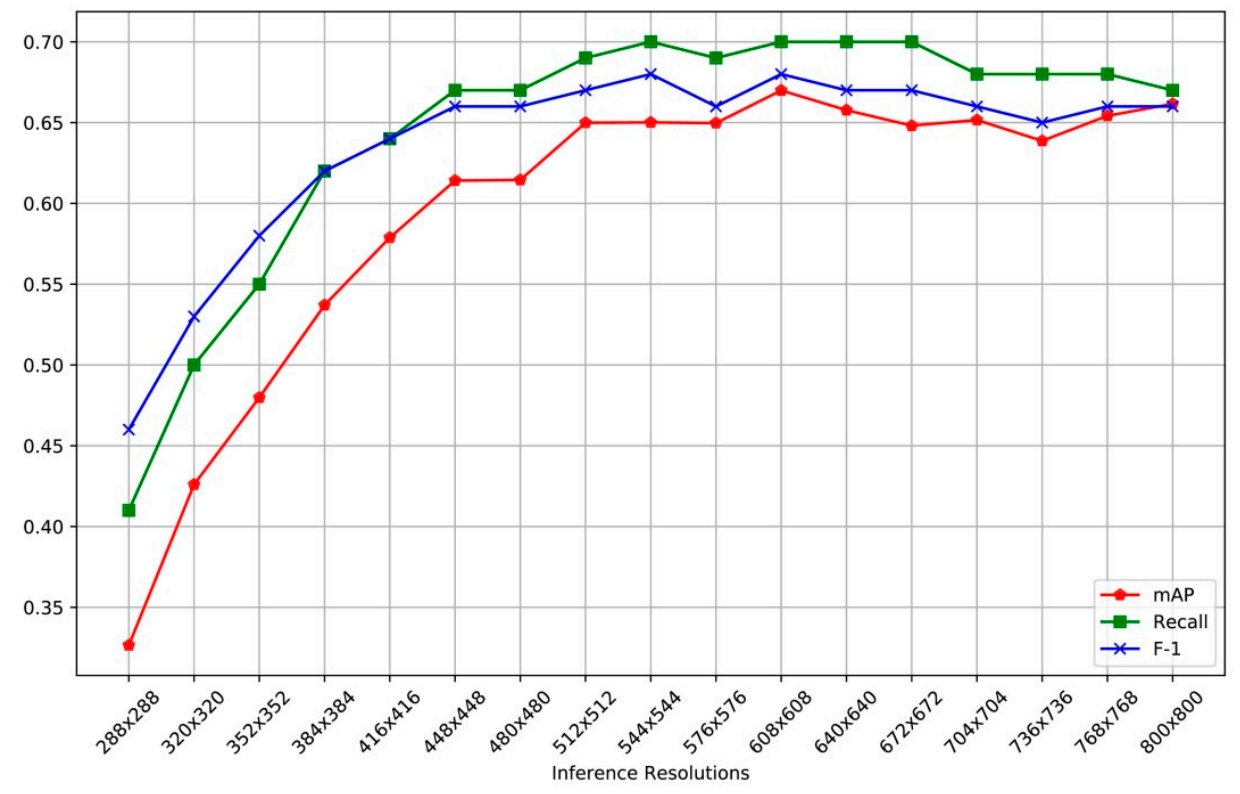

Figure 4. Metrics results for different input resolutions.

Throughout training procedure, the highest model performance was attained with the following network parameter values: Batch size $=64$, momentum $=0.9$, decay $=0.0005$, learning rate $=0.001$, threshold $=0.25$, and iteration number $=45,000$. The detector was able to recognize "swimmers" objects in the dataset with $67 \% \mathrm{mAP}$ and $70 \%$ recall. Given these figures, the detector might seem unreliable in the short term and between consecutive frames, something that does not affect the sought application. With the aim to support our arguments, we provide a link (https://youtu.be/xxAaadmI5GA) containing experimental results from data never experienced by the network during training.

The whole training procedure lasted almost a six-hour run on a Nvidia Tesla V100. The model was validated by assessing the classification accuracy on a class of objects, namely "swimmers". This class consisted of images including open water swimmers (see Figure 5 (left column). Next, once validation and training have been completed, open water swimmer detection tests were performed on real-time videos captured by the rescue $\mathrm{UAV}$, in order to evaluate network accuracy. Results from network testing are as shown in Figure 5 (right column). The model was able to accurately detect and classify the presence of human bodies or shapes in open water. 

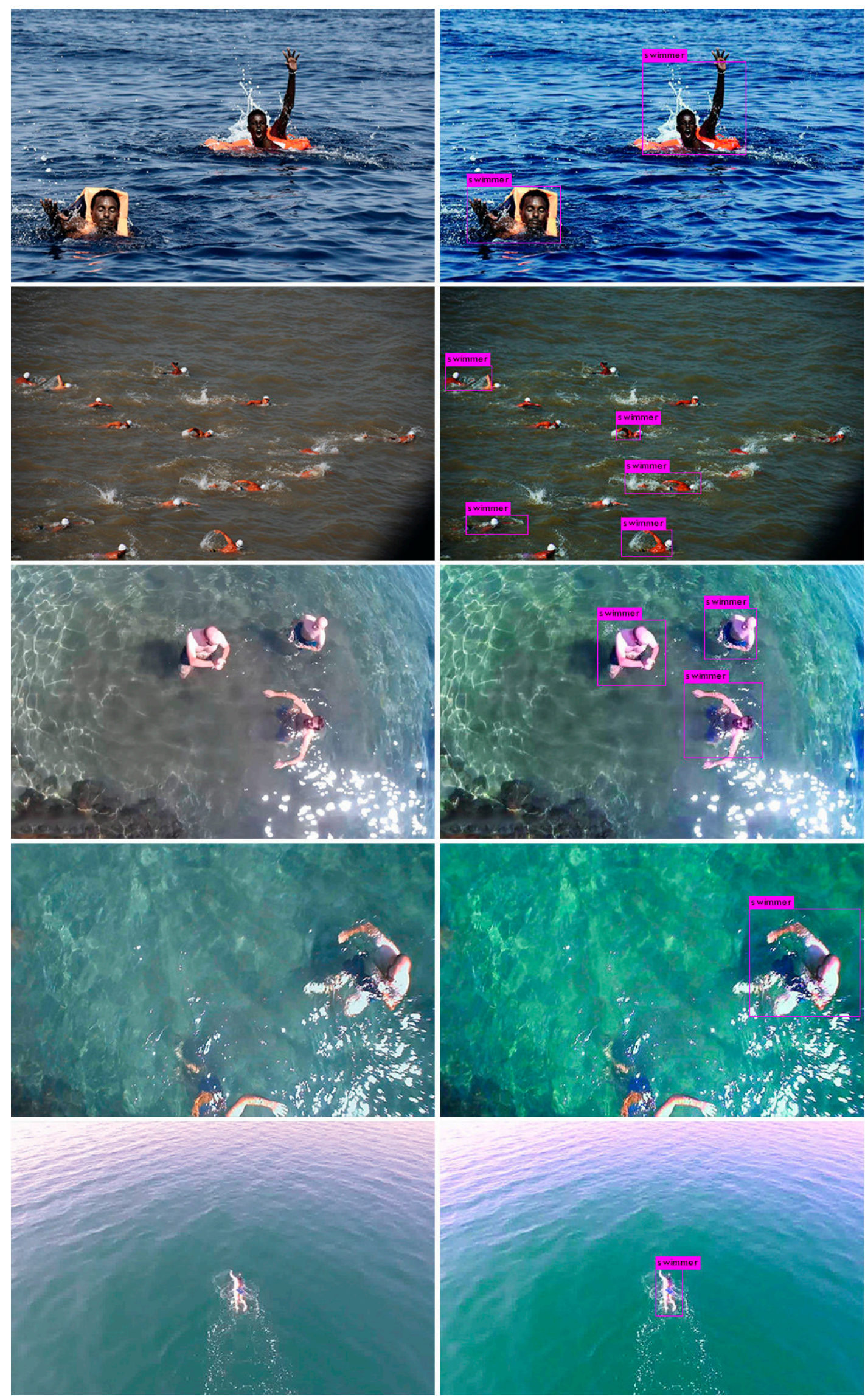

Figure 5. Humans in open waters (left column) before and (right column) after detection procedure. 
In the present work, a Darknet [71] framework was adopted for the detection of both landing targets and humans in peril. Darknet is an open source neural network framework which supports central processing unit (CPU) and graphics processing unit (GPU) computations suitable for a wide variety of tasks relative to computer vision. Darknet is written in C and CUDA and can be easily installed and applied for such tasks due to its extremely large computational power. The main reason for this selection was in order to achieve high-level accuracy as the whole detection procedure had to be implemented with an adequate frame rate due to a swimmer's potential continuous movement. Darknet combined with YOLO provided adequate results due to its high-detection speed and low computation resources for our embedded system.

As an alternative to YOLO, we trained another detector, based on SSD MobileNetV2 [72] architecture with an input image resolution of $300 \times 300$. Using the same training dataset, we used transfer-learning by applying pre-trained weights from the COCO dataset to the model. However, this approach barely provided a mAP of $21 \%$. Thus, it is apparent that YOLO outperforms the MobileNet approach for the given application, so we chose it.

\section{Implementation on the Autonomous UAV}

The primary objective of this project is the integration of machine learning methods to a fully autonomous rescue $\mathrm{UAV}$, for real-time detection of humans in peril, in case of a maritime crisis event occurrence. Towards this end, the first step of this implementation was the application of a vision-based neural network controller for the fully autonomous landing of the rescue UAV on static targets, which was implemented and assessed on a hexacopter bearing an embedded system affording independent take-off, navigation, and landing on a fixed target. The embedded system used for this assessment was a Raspberry $\mathrm{Pi} 3$ and the neural networks' acceleration package was the NNPACK, the combination that provided the adequate effectiveness and accuracy. However, a higher resolution as well as a higher frame rate is needed for the integration of the aforementioned methods to a rescue $U A V$, due to a swimmer's potential continuous movement. For fully autonomous UAV navigation, as well as the automatic detection of human in peril, the detection algorithm should run onboard. Owing to the fact that YOLO calculations are based on a CUDA Toolkit, a powerful GPU embedded system with low consumption would be required. Therefore, for the implementation of the system in this paper, the Nvidia Jetson TX1 was chosen. The algorithm is executed with $12 \mathrm{fps}$, which forms an adequate swimmer's position update rate for the rescue aerial vehicle's prompt performance. The aerial rescue vehicle's technical specifications, used for the implementation of this research, are presented in Table 1 . The fully autonomous rescue UAV and the embedded system for the real-time swimmer detection are depicted in Figure 6.

Table 1. Rescue UAV's technical specifications.

\begin{tabular}{cc}
\hline & Specifications \\
\hline Rotors & 6 \\
Weight & $2700 \mathrm{~g}$ (Excluding Battery) \\
Brushless Motors & $5008-\mathrm{kV} 400(\mathrm{RPM} / \mathrm{V})$ \\
Motors Max. Current & $30 \mathrm{~A}$ \\
Payload Capability & $5 \mathrm{Kg}$ \\
Frame Type & Folding Umbrella \\
Frame Material & Electric Folding \\
Landing Gear & $96 \mathrm{~cm}$ \\
Wheel Base & Lightweight Frame, Portable, Carbon Fiber \\
Battery Type & 1855 High-end Carbon Fiber \\
Rotor Size & $40 \mathrm{~A} / 6 \mathrm{~S}$ \\
Brushless ESC & Goon mAh/30 C \\
Autopilot & GoPro $1920 \times 1080 \mathrm{HD}$ \\
Camera & $1800 \mathrm{~W}$ \\
Hover Power & $25 \mathrm{~min}$ \\
Hover Time (max) &
\end{tabular}




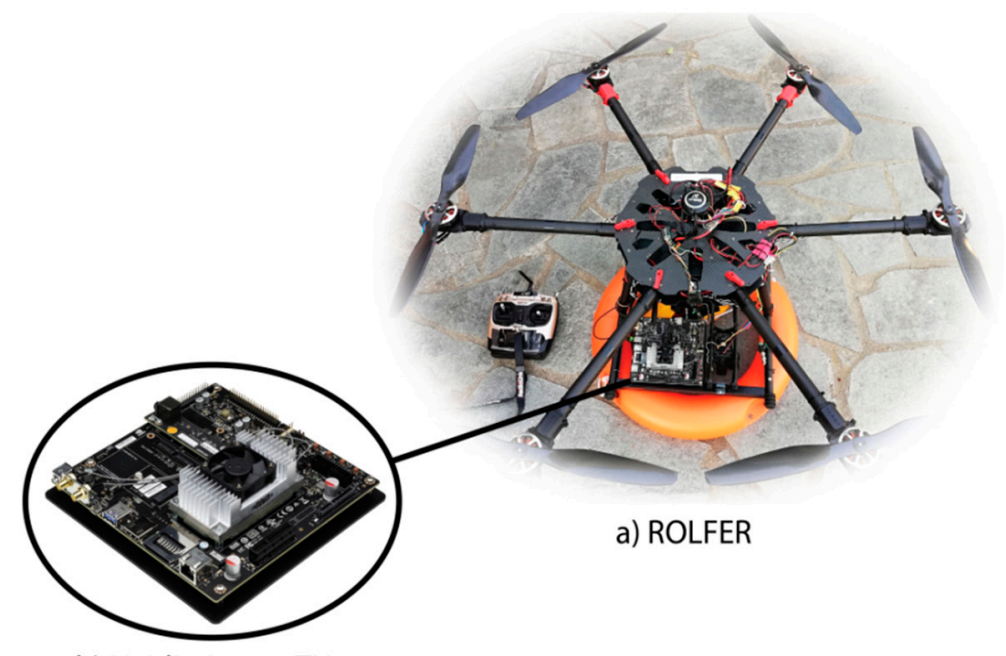

b) Nvidia Jetson TX1

Figure 6. (a) The fully autonomous rescue UAV and (b) the embedded graphics processing unit (GPU) platform Nvidia Jetson TX1.

The entire rescue process of the proposed system, based on the image processing algorithm running on the on-board autonomous computation machine, is illustrated in Figure 7.

The rescue procedure is triggered by a "HELP" message submitted by the SP (Supervised Person-a distressed swimmer being in a supervised area in order to benefit from the proposed system) $[9,10]$. This message is received, validated and decomposed at the ground station (GS) by the main controlling computer. Then, the rescue aerial vehicle's flight-file is generated. The UAV is commanded to automatically arm and take-off. It heads towards the incident area, possibly through one or more intermediate points in order to avoid potential obstacles or restricted regions [10]. Given the limitations of GNSS accuracy in commercially available Android smart watches (ASW) today, the UAV can reach the swimmer with an optimal horizontal position accuracy of $\pm 5 \mathrm{~m}$, with $95 \%$ confidence (also mentioned as 95th percentile). Owing to the complexity of the "GNSS accuracy" definition, a full-length documentary or a several page article would be appropriate to explain such a topic. The testing process for the accuracy of handheld GNSS is conducted under at least two main conditions: (a) an open sky, and (b) a disturbed atmosphere with plenty of obstructions, such as is an urban area with high skyscrapers, which is the worst-case scenario. As we are dealing with open water swimmers or other humans in peril, we assumed that we fall into the first category. Thus, we will mention only the maximum accuracy of GNSS.

For the autonomous rescue UAV to reach the incident area, the UAV's controller checks if the absolute distance between the swimmer and the UAV is less than the pre-specified limits (dlat, dlong) defined by the entire system's administrator during its setup. Details about these limits are analytically described in $[9,10]$. At this point, the potential victim is located within the Field of View (FoV) of the onboard camera, while the UAV flies above a minimum altitude. Provided that our camera's FoV is $82 \mathrm{o}$, the minimum Altitude in order to cover the complete circle of radius $\mathrm{R}=5 \mathrm{~m}$ is about Alt $=$ $5.75 \mathrm{~m}$ (see Figure 8a). Since the UAV is flying at Alt $=10 \mathrm{~m}$, the human in peril will certainly be located inside the cameras' FoV. Then, image processing will be activated and the human detection algorithm will be activated on the on-board autonomous computing machine. A successful human detection process will return the distressed swimmer's coordinates in the image frame and the control algorithm's calculation for the UAV's speed in $x, y$ axes will be activated. Next, the main objective of the UAV motion controller is the aerial vehicle's movement in the proper direction in order to be exactly over the swimmer and to start descending at a pre-specified altitude above him/her. The validation of the UAV's precise positioning is a crucial factor in such rescue systems as the rescue apparatus should be released as close as possible to the distressed human, at a maximum distance of $\pm 0.5 \mathrm{~m}$. 


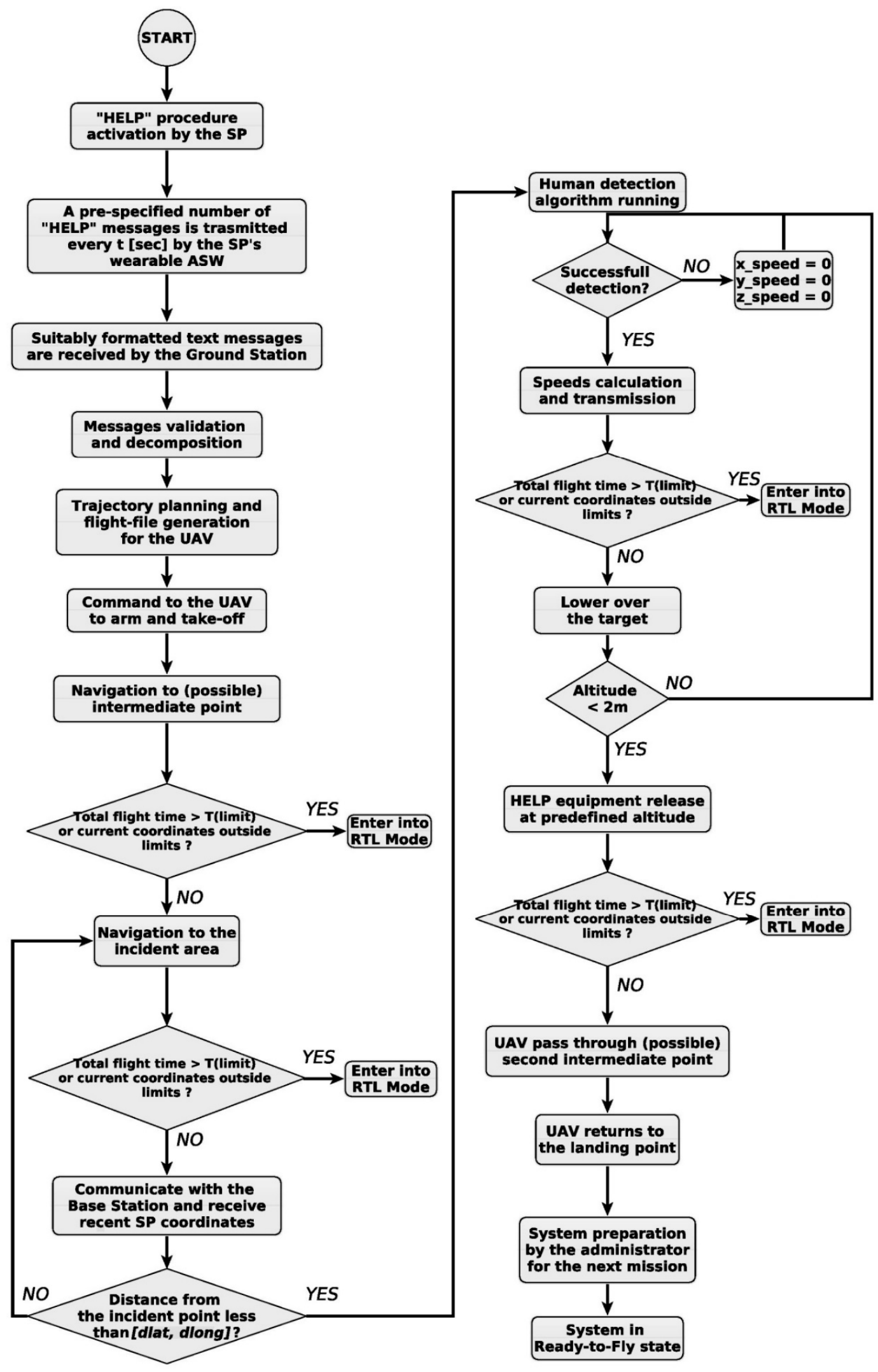

Figure 7. Autonomous Rescue System's Operation Flowchart.

The available data are the camera's resolution and image coordinates of the two opposing angles of the orthogonal box in which the swimmer is located. Image coordinates are defined as the number of pixels of those two points, with axes origin placed at the top left angle $(0,0)$ and the opposing angle 
at $416 \times 416$ image resolution. The UAV's center is defined by the central camera's pixel to $x, y$ axes correspondingly (see Figure $8 b$ ), due to the fact that the camera is placed in the middle of the box, i.e., at

$$
\begin{aligned}
& C_{x}=\frac{416}{2} \\
& C_{y}=\frac{416}{2} .
\end{aligned}
$$

As mentioned above, the main objective is the aerial vehicle's movement in the direction and to the center of the detected swimmer's area.

In order to control the vehicle's speed in 3D space, the desired speed's components $v_{x}, v_{y}, v_{z}$ on each of $x, y, z$ axes, correspondingly, must be calculated and provided to the UAV's controller. Since the $z$-axis speed is the descending speed to the target, it can be defined separately through the UAV control software.

If $\left(t_{x}, t_{y}\right)$ are the coordinates of swimmer's image bounding box centroid (see Figure $\left.8 \mathrm{~b}\right)$ and $\left(c_{x}, c_{y}\right)$ are the UAV's center coordinates, then sides $N, M$ are known, since:

$$
\begin{aligned}
& N=t_{x}-C_{x} \\
& M=t_{y}-C_{y}
\end{aligned}
$$

Thus

$$
\text { dist }=\sqrt{N^{2}+M^{2}}
$$

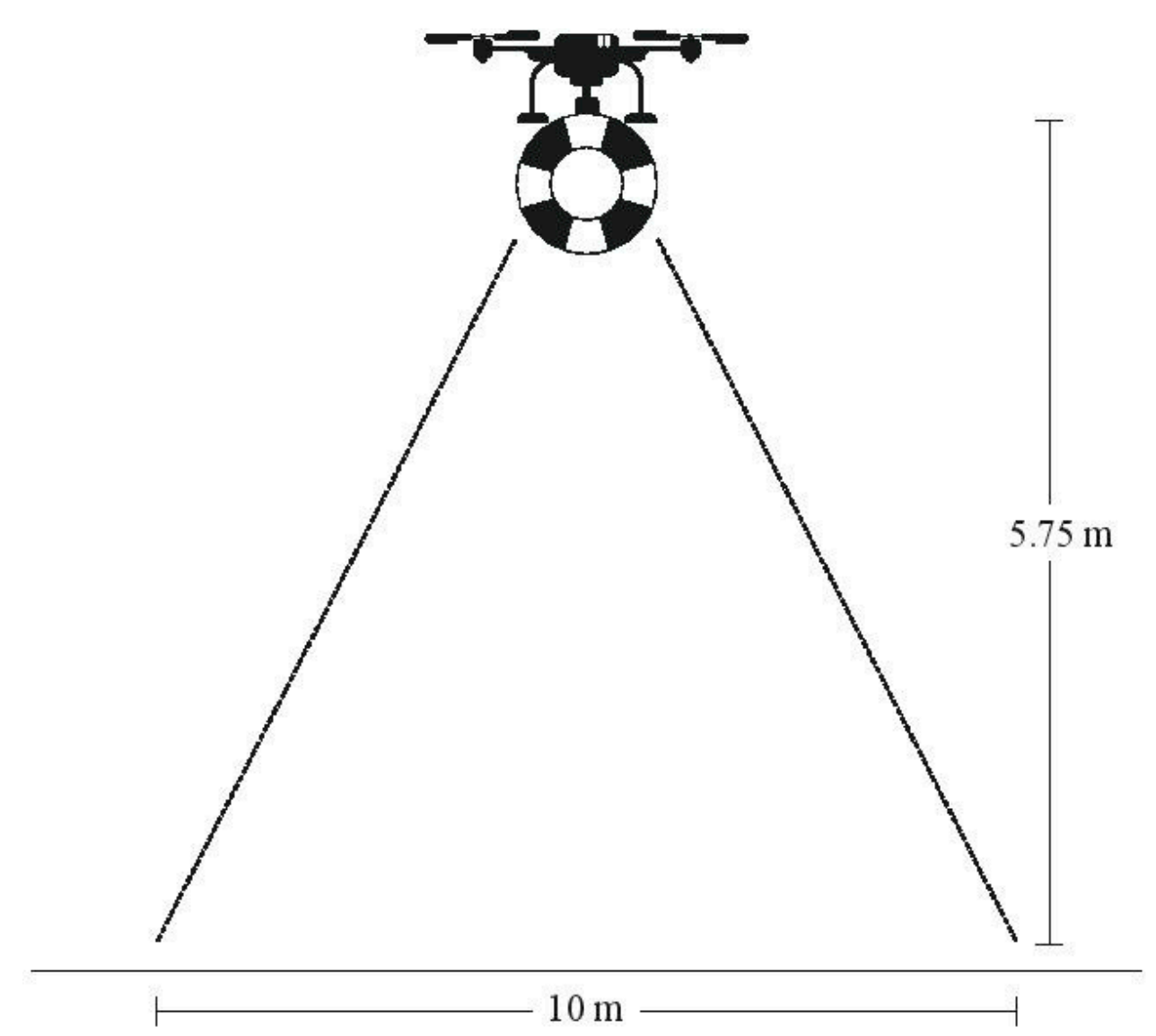

(a)

Figure 8. Cont. 


\section{$(0,0)$}

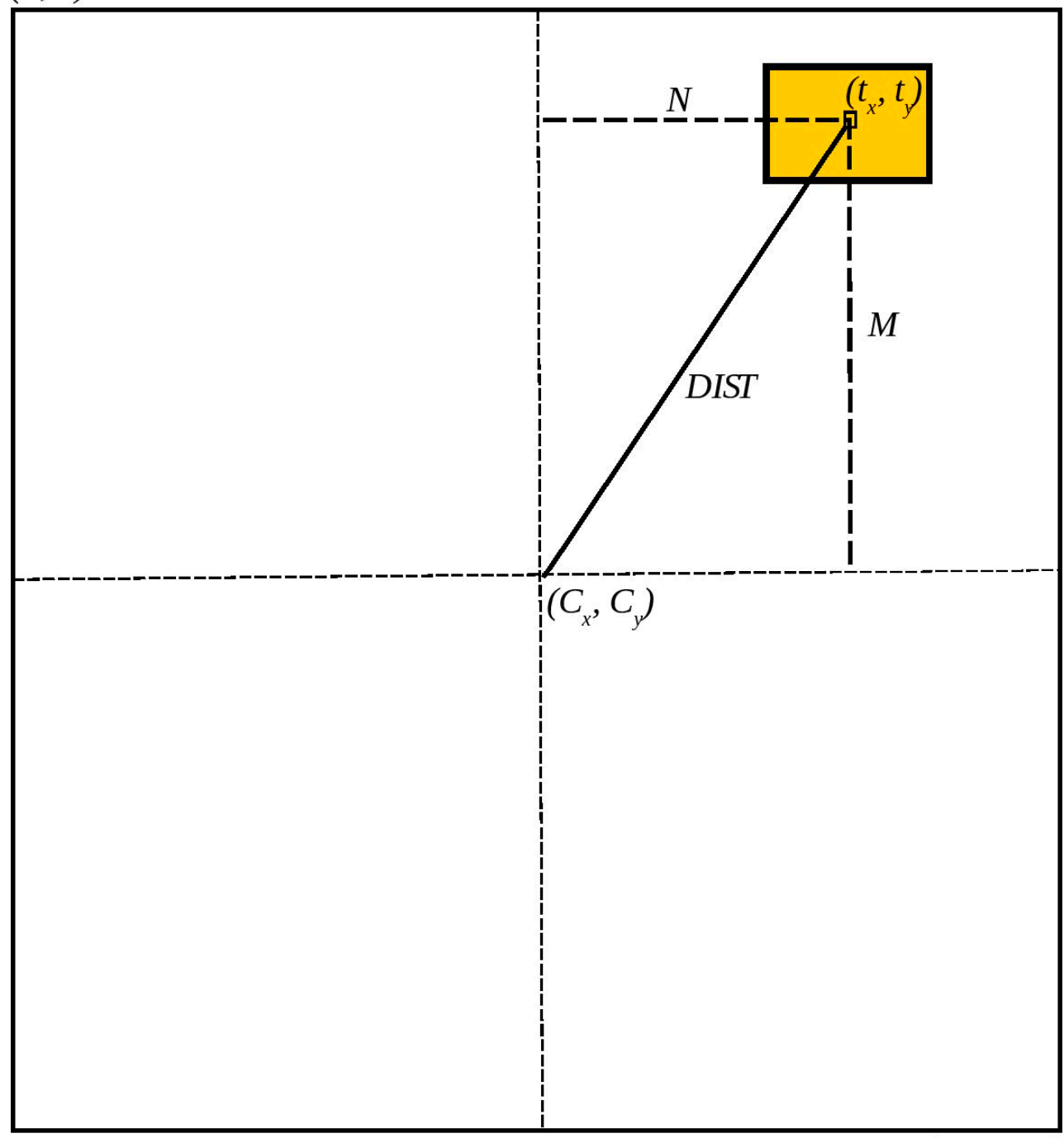

(b)

$(416,416)$

Figure 8. Rescue UAV's camera field of view (FoV) (a) The proportion between sides $N, M$ and (b) speeds $t_{x}, t_{y}$ on $x, y$ axes.

From Equations (4)-(6), it is obvious that the direction of the vector of resultant speed is equal to the direction of the vector of the hypotenuse dist, resulting from the proportion ratio between the sides $N, M$ and the speeds on $x, y$ axes.

Given the proportionalities, we can further derive that:

$$
\begin{aligned}
& \frac{\text { speed }_{y}}{N}=\frac{\text { total }_{\text {speed }}}{\text { dist }} \\
& \frac{\text { speed }_{x}}{M}=\frac{\text { total }_{\text {speed }}}{\text { dist }}
\end{aligned}
$$

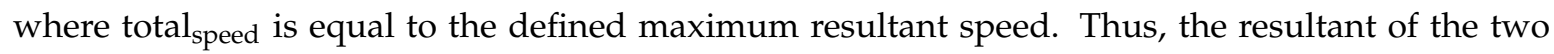
individual speeds, directs the hexacopter towards the swimmer.

Upon the precise detection of the distressed human, the autonomous rescue hexacopter descends at a system's administrator-defined altitude above him/her and the rescue apparatus is released. After that action, the hexacopter returns to the shore. Figure 9 depicts the simulation results for the expected 
displacement of a released ring with $\mathrm{R}=25 \mathrm{~cm}$ diameter and an elliptical intersection of $10 \times 8 \mathrm{~cm}$ $(a=5 x, b=4)$, under the action of side-wind.

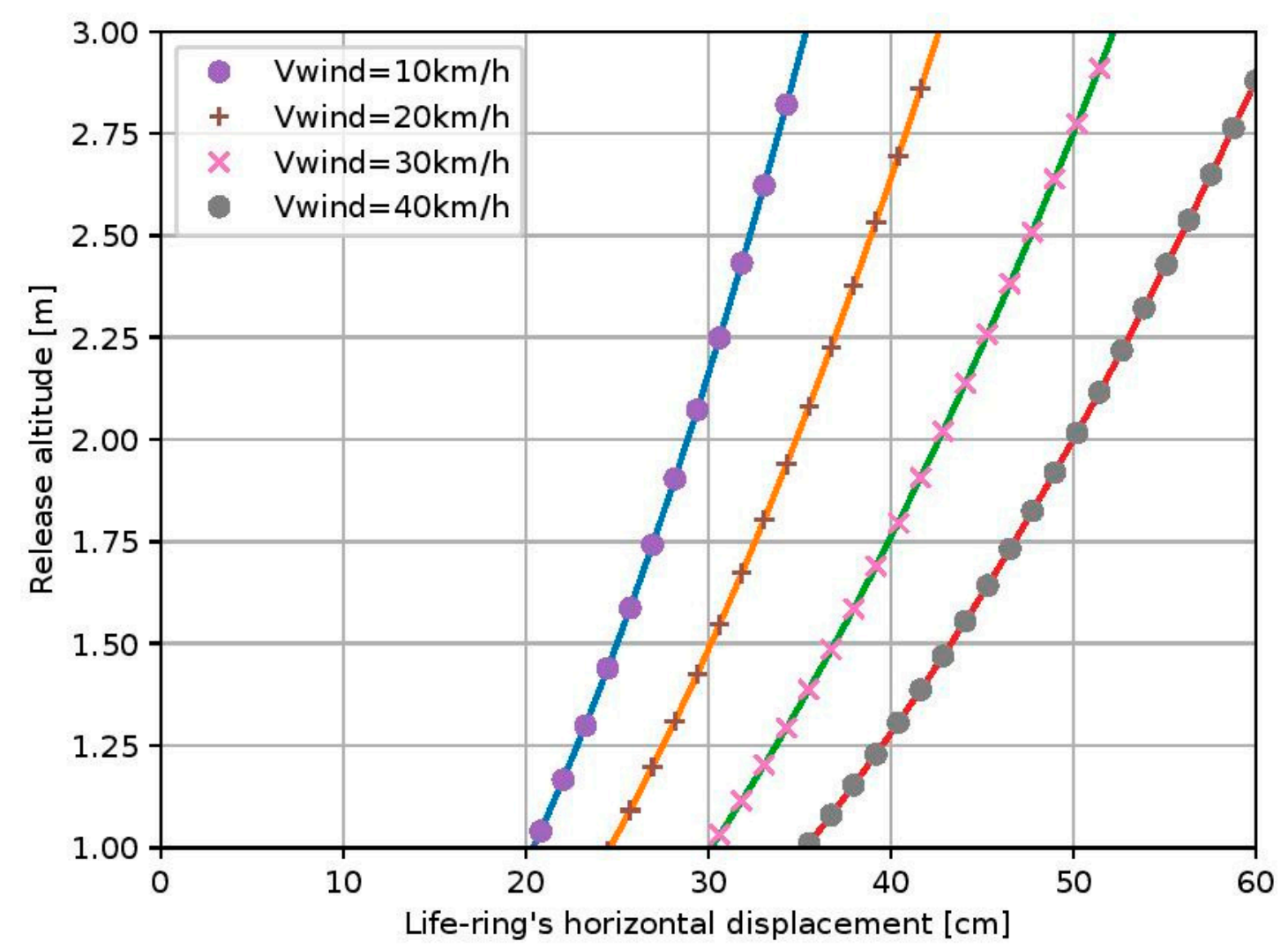

Figure 9. The life-ring's horizontal displacement related to the UAV's release altitude under several wind speeds.

Results of the life-ring's release accuracy were calculated after simulating the whole rescue apparatus release process. Air flow velocity is considered as a random variable. Bearing in mind that air resistance is proportional to the square of falling velocity, the life-ring's equation of motion can be expressed as

$$
m \frac{d v}{d t}=m g-\frac{1}{2} \rho C_{d} A v^{2}
$$

where:

$m$ is the life-ring's mass $(K g)$,

$A$ is the life-ring's horizontal cross-sectional area $\left(\mathrm{m}^{2}\right)$,

$P$ is the air density $\left(\mathrm{kg} / \mathrm{m}^{3}\right)$,

$C_{D}$ is the drag coefficient,

The horizontal force acting on the life-ring in a fluid flow can be expressed as

$$
F_{L}=C_{L} F_{w}=C_{D} \frac{1}{2} \rho v_{w}^{2} A_{1}
$$

where:

$F_{L}$ is the horizontal force $(N)$,

$C_{L}$ is the lifting coefficient

$F_{w}$ is the wind force $(N)$

$A_{1}$ is the vertical life-ring's cross-sectional area $\left(\mathrm{m}^{2}\right)$ 
As a random variable, the horizontal force can act on the life-ring in any direction. However, we assumed the worst-case scenario where this force acts on the horizontal direction during the life-ring's fall down.

Taking into account the UAV's technical specifications, the maximum allowable wind-speed limit for safe flights is $5 \mathrm{Bf}(28-39 \mathrm{~km} / \mathrm{h})$ and hence we can estimate the life-ring's maximum horizontal displacement under several horizontal wind speeds. According to Figure 9, in order to stay within the limits of $\pm 0.5 \mathrm{~m}$ accuracy, in a worst-case scenario of $40 \mathrm{~km} / \mathrm{h}$ side-wind, the life-ring must be released from a maximum altitude of $2 \mathrm{~m}$. This can be considered as a safe distance, in the sense that it does not prevent a human in peril from grasping the life preserver.

Finally, the aerial vehicle follows the reverse trajectory and automatically returns to the landing area. Then, the system is prepared for the next rescue mission by its administrator and gets in ready-to-fly state.

\section{Conclusions}

The combination of UAV technologies and real-time computer vision with deep learning techniques for search and rescue purposes has been presented in this work. A novel rescue platform for the instantaneous detection and rescue of humans in peril has been integrated. Based-on state-of-the-art human detection techniques, the proposed system is capable of precisely detecting and rescuing open water swimmers in peril, without apparent human intervention. Distressed swimmer detection is performed on-board a fully autonomous rescue UAV, capable of executing time-crucial life-saving operations in a fully unsupervised manner.

Hardware and software configurations for such implementation have also been outlined. The novelty of this implementation banks on the combination of GNSS techniques and computer vision algorithms. The model used in this research was a CNN with Tiny YOLOv3 architecture running on the embedded autonomous computing machine NVIDIA Jetson X1. Training, validation, and testing processes have also been presented. Lastly, due to the restricted amount of training dataset available online, the authors obtained their own aerial images for the training dataset from the proposed aerial rescue UAV. Real flights evaluation revealed the superiority of the adopted deep learning techniques. Our model's attained mAP was in the order of $67 \%$, which is a very adequate figure for an on-board human detection process. Moreover, simulation results for the rescue apparatus fall down accuracy are presented.

The onboard image processing hardware, using the Nvidia Jetson TX1, offers the capability of real-time processing, thus avoiding the transmission of the video sequence to a ground station for processing and returning the results to the UAV, something that could lead to undesirable and crucial time delays.

While the proposed rescue system has been implemented for open water swimmer detection, it would be capable of detecting humans and providing emergency services to people practicing winter sport activities, with only a few modifications. Based on the high level of the achieved accuracy for both detection and classification, there are limitless opportunities for the proposed approach to be applied in SAR missions in numerous terrains or environments.

Author Contributions: E.L. designed and implemented the whole rescue system and wrote and edited the paper. N.S. and A.T. developed the detection and identification algorithms of the image processing system and wrote the respective section. All these three authors contributed to perform the experiments, obtain their own dataset of aerial images as well as processing them. K.T. and A.M. had the initial idea for the implementation of this project and the final revision of the paper with A.G. who supervised and guided the whole project.

Funding: This research received no external funding.

Conflicts of Interest: The authors declare no conflicts of interest. 


\section{Abbreviations}

The following abbreviations are used in this manuscript:

$\begin{array}{ll}\text { UAVs } & \text { Unmanned Aerial Vehicles } \\ \text { SAR } & \text { Search and Rescue } \\ \text { GNSS } & \text { Global Navigation Satelite System } \\ \text { ROLFER } & \text { Robotic Lifeguard For Emergency Rescue } \\ \text { PPP } & \text { Precise Point Positioning } \\ \text { CNNs } & \text { Convolutional Neural Networks } \\ \text { T-CNNs } & \text { Temporal Convolutional Neural Networks } \\ \text { mAP } & \text { mean Average Precision } \\ \text { UAS } & \text { Unmanned Aerial System } \\ \text { SVM } & \text { Support Vector Machine } \\ \text { FCNN } & \text { Fully Convolutional Neural Network } \\ \text { IoU } & \text { Intersection Over Union } \\ \text { CPU } & \text { Central Processing Unit } \\ \text { GPU } & \text { Graphics Processing Unit } \\ \text { FPS } & \text { Frames Per Second } \\ \text { SP } & \text { Supervised Person } \\ \text { GS } & \text { Ground Station } \\ \text { ASW } & \text { Android Smart Watch } \\ \text { FOV } & \text { Field Of View }\end{array}$

\section{References}

1. Valavanis, K.; Vachtsevanos, G. UAV Applications. In Handbook of Unmanned Aerial Vehicles; Valavanis, K., Vachtsevanos, G., Eds.; Springer: Berlin, Germany, 2015; Volume 3, pp. 2639-2641. [CrossRef]

2. De Cubber, G.; Doroftei, D.; Rudin, K.; Berns, K.; Matos, A.; Serrano, D.; Sanchez, J.; Govindaraj, S.; Bedkowski, J.; Roda, R.; et al. Introduction to the use of robotic tools for search and rescue. In Search and Rescue Robotics-From Theory to Practice; IntechOpen: London, UK, 2017; pp. 1-17. [CrossRef]

3. Polka, M.; Ptak, S.; Kuziora, L.; Kuczynska, A. The use of unmanned aerial vehicles by urban search and rescue groups. In Drones-Applications; Dekoulis, G., Ed.; IntechOpen: London, UK, 2017; pp. 84-95. [CrossRef]

4. Marques, M.M.; Lobo, V.; Batista, R.; Oliveira, J.; Aguiar, A.P.; Silva, J.E.; de Sousa, J.B.; Nunes, M.D.F.; Ribeiro, R.A.; Bernardino, A.; et al. An unmanned aircraft system for maritime operations: The sense and avoid subsystem with software-in-the-loop evaluation. Int. J. Adv. Robot. Syst. 2018, 15. [CrossRef]

5. Seguin, C.; Blaquière, G.; Loundou, A.; Michelet, P.; Markarian, T. Unmanned aerial vehicles (drones) to prevent drowning. Resuscitation 2018, 127, 63-67. [CrossRef] [PubMed]

6. Tomotani, J. Using unmanned aerial vehicles in search operations. J. Geek Stud. 2015, 2, 41-53.

7. Yeong, S.P.; King, L.M.; Dol, S.S. A review on marine search and rescue operations using unmanned aerial vehicles. Int. J. Mech. Aerosp. Ind. Mech. Manuf. Eng. 2015, 9, 396-399.

8. Grogan, S.; Pellerin, R.; Gamache, M. The use of unmanned aerial vehicles and drones in search and rescue operations-A survey. In Proceedings of the PROLOG 2018-the 4th Edition at the Logistics Institute, Hull, UK, 28-29 June 2018.

9. Lygouras, E.; Gasteratos, A.; Tarchanidis, K. ROLFER: An innovative proactive platform to reserve swimmer's safety. In Proceedings of the 4th International Conference on Information Systems for Crisis Response and Management in Mediterranean Countries (ISCRAMed 2017), Xanthi, Greece, 18-20 October 2017.

10. Lygouras, E.; Gasteratos, A.; Tarchanidis, K.; Mitropoulos, A. ROLFER: A fully autonomous aerial rescue support system. Microprocess. Microsyst. 2018, 61, 32-42. [CrossRef]

11. Chen, B.; Gao, C.; Liu, Y.; Sun, P. Real-time precise point positioning with a Xiaomi MI 8 android smartphone. Sensors 2019, 19, 2835. [CrossRef] [PubMed]

12. Robustelli, U.; Baiocchi, V.; Pugliano, G. Assessment of dual frequency GNSS observations from a Xiaomi Mi 8 android smartphone and positioning performance analysis. Electronics 2019, 8, 91. [CrossRef] 
13. Elmezayen, A.; El-Rabbany, A. Precise Point Positioning Using World's First Dual-Frequency GPS/GALILEO Smartphone. Sensors 2019, 19, 2593. [CrossRef]

14. LeCun, Y.; Bengio, Y.; Hinton, G. Deep Learning. Nature 2015, 521, 436-444. [CrossRef]

15. Deng, L.; Yu, D. Deep Learning: Methods and applications. Found. Trends Signal Process. 2014, 7, $197-387$. [CrossRef]

16. Lea, C.; Flynn, M.; Vidal, R.; Reiter, A.; Hager, G. Temporal convolutional networks for action segmentation and detection. In Proceedings of the IEEE Conference on Computer Vision and Pattern Recognition (CVPR), Las Vegas, NV, USA, 26 June-1 July 2016.

17. Sabu, E.; Suresh, K. Object detection from video using temporal convolutional network. In Proceedings of the IEEE Recent Advances in Intelligent Computational Systems (RAICS), Trivandrum, India, 6-8 December 2018.

18. Nair, N.; Thomas, C.; Jayagopi, D. Human activity recognition using temporal convolutional network. In Proceedings of the 5th international Workshop on Sensor-Based Activity Recognition and Interaction (iWOAR '18), Berlin, Germany, 20-21 September 2018.

19. Zhongang, C.; Cunjun, Y.; Quang-Cuong, P. 3D Convolution on RGB-D point clouds for accurate model-free object pose estimation. arXiv 2018, arXiv:1812.11284.

20. Rui, H.; Chen, C.; Mubarak, S. An end-to-end 3D convolutional neural network for action detection and segmentation in videos. arXiv 2017, arXiv:1712.01111.

21. Ji, S.; Xu, W.; Yang, M.; Yu, K. 3D convolutional neural networks for human action recognition. IEEE Trans. Pattern Anal. Mach. Intell. 2010, 35, 495-502. [CrossRef]

22. Carrio, A.; Sampedro, C.; Rodriguez-Ramos, A.; Campoy, P. A review of deep learning methods and applications for unmanned aerial vehicles. J. Sens. 2017, 2017, 1-13. [CrossRef]

23. Radovic, M.; Adarkwa, O.; Wang, Q. Object recognition in aerial images using convolutional neural networks. J. Imaging 2017, 3, 21. [CrossRef]

24. Kyrkou, C.; Plastiras, G.; Theocharides, T.; Venieris, S.; Bouganis, C. DroNet: Efficient convolutional neural network detector for real-time UAV applications. In Proceedings of the IEEE 2018 Design, Automation \& Test in Europe Conference \& Exhibition (DATE), Dresden, Germany, 19-23 March 2018.

25. Hongguang, L.; Yang, S.; Baochang, Z.; Yufeng, W. Superpixel-based feature for aerial image scene recognition. Sensors 2018, 18, 156. [CrossRef]

26. Santos, N.; Lobo, V.; Bernardino, A. 3D model-based estimation for UAV tracking. In Proceedings of the OCEANS, 2018 MTS/IEEE, Charleston, SC, USA, 22-25 October 2018.

27. Babis, L.; Karakasis, E.; Amanatiadis, A.; Gasteratos, A. Can speedup assist accuracy? An on-board GPU-accelerated image georeference method for UAVs. In Proceedings of the 10th International Conference on Computer Vision Systems (ICVS 2015), Copenhagen, Denmark, 6-9 July 2015.

28. Giitsidis, T.; Karakasis, E.; Gasteratos, A.; Sirakoulis, G. Human and fire detection from high altitude UAV images. In Proceedings of the 23rd Euromicro International Conference on Parallel, Distributed, and Network-Based Processing, Turku, Finland, 4-6 March 2015.

29. Amanatiadis, A.; Karakasis, E.G.; Bampis, L.; Giitsidis, T.; Panagiotou, P.; Sirakoulis, G.C.; Gasteratos, A.; Tsalides, P.; Goulas, A.; Yakinthos, K. The HCUAV project: Electronics and software development for medium altitude remote sensing. In Proceedings of the 12th IEEE International Symposium on Safety, Security, and Rescue Robotics, Hokkaido, Japan, 27-30 October 2014.

30. Saponara, S. Sensing and connection systems for assisted and autonomous driving and unmanned vehicles. Sensors 2018, 18, 1999. [CrossRef]

31. Petritoli, E.; Leccese, F.; Ciani, L. Reliability and maintenance analysis of unmanned aerial vehicles. Sensors 2018, 18, 3171. [CrossRef]

32. Konovalenko, I.; Kuznetsova, E.; Miller, A.; Miller, B.; Popov, A.; Shepelev, D.; Stepanyan, K. New approaches to the integration of navigation systems for autonomous unmanned vehicles (UAV). Sensors 2018, 18, 3010. [CrossRef]

33. Chongyang, L.; Yalin, D.; Ming, Z.; Jihong, X.; Mengyang, L.; Qihui, L. Vehicle detection in aerial images using a fast oriented region search and the vector of locally aggregated descriptors. Sensors 2019, 19, 3294. [CrossRef]

34. Xiang, X.; Zhai, M.; Lv, N.; El Saddik, A. Vehicle counting based on vehicle detection and tracking from aerial videos. Sensors 2018, 18, 2560. [CrossRef] 
35. Saqib, M.; Khan, S.; Sharma, N.; Blumenstein, M. A study on detecting drones using deep convolutional neural networks. In Proceedings of the 14th IEEE International Conference on Advanced Video and Signal Based Surveillance (AVSS), Lecce, Italy, 29 August-1 September 2017.

36. Opromolla, R.; Fasano, G.; Accardo, D. A vision-based approach to UAV detection and tracking in cooperative applications. Sensors 2018, 18, 3391. [CrossRef]

37. Maher, A.; Taha, H.; Zhang, B. Realtime multi-aircraft tracking in aerial scene with deep orientation. J. Real-Time Image Process. 2018, 15, 495-507. [CrossRef]

38. Farlik, J.; Kratky, M.; Casar, J.; Stary, V. Multispectral detection of commercial unmanned aerial vehicles. Sensors 2019, 19, 1517. [CrossRef]

39. Jin, R.; Jiang, J.; Qi, Y.; Lin, D.; Song, T. Drone detection and pose estimation using relational graph networks. Sensors 2019, 19, 1479. [CrossRef]

40. Ichim, L.; Popescu, D. Road detection and segmentation from aerial images using a CNN based system. In Proceedings of the 41st International Conference on Telecommunications and Signal Processing (TSP), Athens, Greece, 4-6 July 2018.

41. Boonpook, W.; Tan, Y.; Ye, Y.; Torteeka, P.; Torsri, K.; Dong, S. A deep learning approach on building detection from unmanned aerial vehicle-based images in riverbank monitoring. Sensors 2018, 18, 3921. [CrossRef]

42. Song, W.; Zhong, B.; Sun, X. Building corner detection in aerial images with fully convolutional networks. Sensors 2019, 19, 1915. [CrossRef]

43. Byunghyun, K.; Soojin, C. Automated vision-based detection of cracks on concrete surfaces using a deep learning technique. Sensors 2018, 18, 3452. [CrossRef]

44. Suk-Ju, H.; Yunhyeok, H.; Sang-Yeon, K.; Ah-Yeong, L.; Ghiseok, K. Application of deep-learning methods to bird detection using unmanned aerial vehicle imagery. Sensors 2019, 19, 1651. [CrossRef]

45. Rivas, A.; Chamoso, P.; González-Briones, A.; Corchado, J. Detection of cattle using drones and convolutional neural networks. Sensors 2018, 18, 2048. [CrossRef]

46. Dang, L.; Hassan, S.; Suhyeon, I.; Sangaiah, A.; Mehmood, I.; Rho, S.; Seo, S.; Moon, H. UAV based wilt detection system via convolutional neural networks. Sustain. Comput. Inform. Syst. 2018, 2018. [CrossRef]

47. Tayara, H.; Chong, K. Object detection in very high-resolution aerial images using one-stage densely connected feature pyramid network. Sensors 2018, 18, 3341. [CrossRef]

48. Din, A.; Bona, B.; Morrissette, J.; Hussain, M.; Violante, M.; Naseem, F. Embedded low power controller for autonomous landing of UAV using artificial neural network. In Proceedings of the FIT: 2012 10th International Conference on Frontiers of Information Technology, Islamabad, Pakistan, 17-19 December 2012.

49. Falanga, D.; Zanchettin, A.; Simovic, A.; Delmerico, J.; Scaramucca, D. Vision-based autonomous quadrotor landing on a moving platform. In Proceedings of the 15th IEEE International Symposium on Safety, Security and Rescue Robotics (SSRR), Shanghai, China, 11-13 October 2017.

50. Alenezi, M.; Almeshal, A. A Vision-based neural network controller for the autonomous landing of a quadrotor on moving targets. Robotics 2018, 7, 71. [CrossRef]

51. Adam, A.; Elmaleeh, M.; Mahmoud, D. A smart neural network based algorithm for landing control of autonomous unmanned aerial vehicle. Int. J. Adv. Res. Sci. Eng. 2017, 6, 1175-1188.

52. AlDahoul, N.; Sabri, A.; Mansoor, A. Real-time human detection for aerial captured video sequences via deep models. Comput. Intell. Neurosci. 2018, 2018, 1639561. [CrossRef]

53. Nikouei, S.; Chen, Y.; Song, S.; Xu, R.; Choi, B.; Faughnan, T. Real-time human detection as an edge service enabled by a lightweight CNN. In Proceedings of the IEEE International Conference on Edge Computing (EDGE), San Francisco, CA, USA, 2-7 July 2018.

54. De Oliveira, D.; Wehrmeister, M. Using deep learning and low-cost RGB and thermal cameras to detect pedestrians in aerial images captured by multirotor UAV. Sensors 2018, 18, 2244. [CrossRef]

55. Tzelepi, M.; Tefas, A. Human crowd detection for drone flight safety using convolutional neural networks. In Proceedings of the 25th European Signal Processing Conference (EUSIPCO), Kos, Greece, 28 August-2 September 2017.

56. Awad, F.; Shamroukh, R. Human detection by robotic urban search and rescue using image processing and neural networks. Int. J. Intell. Sci. 2014, 4, 39-53. [CrossRef]

57. Bejiga, M.; Zeggada, A.; Nouffidj, A.; Melgani, F. A convolutional neural network approach for assisting avalanche search and rescue operations with UAVs imagery. Remote Sens. 2017, 9, 100. [CrossRef] 
58. Gabrlik, P.; Janata, P.; Zalud, L.; Harcarik, J. Towards automatic UAS-based snow-field monitoring for microclimate research. Sensors 2019, 19, 1945. [CrossRef]

59. Tijtgat, N.; Van Ranst, W.; Volckaert, B.; Goedeme, T.; De Turck, F. Embedded Real-Time Object Detection for a UAV Warning System. In Proceedings of the IEEE International Conference on Computer Vision Workshops (ICCVW), Venice, Italy, 22-29 October 2017.

60. Al-Kaff, A.; Gómez-Silva, M.J.; Moreno, F.M.; de la Escalera, A.; Armingol, J.M. An appearance-based tracking algorithm for aerial search and rescue purposes. Sensors 2019, 19, 652. [CrossRef]

61. Hrabia, C.; Hessler, A.; Xu, Y.; Seibert, J.; Brehmer, J.; Albayrak, S. EffFeu project: Towards mission-guided application of drones in safety and security environments. Sensors 2019, 19, 973. [CrossRef]

62. Pham, H.; La, H.; Seifer, D.; Nguyen, L. Reinforcement learning for UAVs autonomous navigation. In Proceedings of the 16th IEEE International Symposium on Safety, Security and Rescue Robotics (SSRR), Philadelphia, PA, USA, 6-8 August 2018.

63. Niroui, F.; Zhang, K.; Kashino, Z.; Nejat, G. Deep reinforcement learning robot for search and Rescue operations: Exploration in unknown cluttered environments. IEEE Robot. Autom. Mag. 2019, 4, 610-617. [CrossRef]

64. Sampredo, C.; Rodriguez-Ramos, A.; Bavle, H.; Carrio, A.; Puente, P.; Campoy, P. A fully autonomous aerial robot for search and rescue applications in indoor environments using learning- based techniques. J. Int. Robot. Syst. 2018, 2018, 1-27. [CrossRef]

65. Nielsen, M. Neural Networks and Deep Learning. Available online: http://neuralnetworksanddeeplearning. com (accessed on 15 July 2019).

66. Zeiler, M.D.; Fergus, R. Visualizing and understanding convolutional networks. In Proceedings of the 13th European Conference on Computer Vision (EECV 2014), Zurich, Switzerland, 6-12 September 2014.

67. Redmon, J.; Divvala, S.; Girshick, R.; Farhadi, A. You only look once: Unified, real-time object detection. arXiv 2016, arXiv:1506.02640.

68. Redmon, J.; Farhadi, A. Yolo9000: Better, faster, stronger. arXiv 2016, arXiv:1612.08242.

69. Redmon, J.; Farhadi, A. YOLOv3: An Incremental Improvement. arXiv 2016, arXiv:1804.02767.

70. Lin, T.Y.; Maire, M.; Belongie, S.; Hays, J.; Perona, P.; Ramanan, D.; Dollár, P.; Zitnick, C.L. Microsoft COCO: Common objects in context. arXiv 2014, arXiv:1405.0312.

71. Redmon, J. Darknet: Open Source Neural Networks in c. Available online: http://pjreddie.com/darknet/ (accessed on 15 July 2019).

72. Sandler, M.; Howard, A.; Zhu, M.; Zhmoginov, A.; Chen, L. MobileNetV2: Inverted residuals and linear bottlenecks. In Proceedings of the IEEE/CVF Conference on Computer Vision and Pattern Recognition (CVPR), Salt Lake City, UT, USA, 18-22 June 2018. 